\title{
LIST OF COLORED PHOTOGRAPHS
}

Many of the specimens used to produce the accompanying colored photographs were collected by various individuals at my request. For those who participated in this project acknowledgments are here made.

With the exception of the colored photograph of Hall scale, Nilotaspis halli (Green) which has been enlarged three diameters $(\times 3)$, the remainder have been photographed actual size on the infested plant part. It is hoped these colored photographs will help in identifying a few of our more common California diaspidids as they are seen in the field.

Plate 1 and the top row of plate 2 include those diaspidids belonging to the tribe Aspidiotini; the remainder are referable to the tribe Diaspidini. The species have not been arranged chronologically under each tribe, but instead are assembled more for the purpose of eye appeal and beauty.

The following list includes specific collection data for each photograph:

\section{PLATE 1}

Aonidiella aurantii (Maskell), Pomona, Los Angeles Co., December 26, 1953, on Citrus limon (lemon) (H. L. McKenzie).

Aonidiella citrina (Coquillett), Reedley Area, Fresno Co., January, 1954, on Citrus sinensis (orange) (T. E. Corn).

Aspidiotus hederae (Vallot), Sacramento, November, 1953, on Hedera helix (ivy) (R. Z. Rollins).

Chrysomphalus bifasciculatus Ferris, Sacramento, November, 1953, on Hedera helix (ivy) (R. Z. Rollins).

Diaspidiotus ancylus (Putnam), Hemet, Riverside Co., June 28, 1954, on Prunus armeniaca (apricot) (H. L. McKenzie).

Hemiberlesia degenerata (Leonardi), Sanger, Fresno Co., May 13, 1954, on Camellia sp. (J. C. Bedford).

Hemiberlesia lataniae (Signoret), Orange Co., May 30, 1954, on Persea sp. (avocado) (D. Bishop).

Lindingaspis rossi (Maskell), Montecito, Santa Barbara Co., December 6, 1954, on Sequoia sempervirens (redwood) and Araucaria sp. (W. S. Cummings).

Nuculaspis californica (Coleman), Lake Gregory, San Bernardino Co., March 9, 1954, on Pinus ponderosa (yellow pine) (G. L. Downing).
PLATE 2

Aonidomytilus ceanothi Ferris, Larson Valley, El Dorado Co., December 27, 1954, on Ceanothus integerrimus (L. Mobley).

Aulacaspis rosae (Bouché), Sacramento, May 28, 1954, on Rubus sp. (mammoth blackberry) (H. H. Keifer).

Carulaspis visci (Schrank), Sacramento, May 26, 1954, on Juniperus sp. (juniper) (H. L. McKenzie).

Diaspis bromeliae (Kerner), San Diego, February 23, 1954, on Bromeliad (G. Hill and R. J. Buckner).

Diaspis echinocacti (Bouché), Norco, Riverside Co., June 28, 1954, on Opuntia hamiltoniae (cactus) (R. M. Hawthorne and H. L. McKenzie).

Diaspis manzanitae (Whitney), Magalia, Butte Co., October go, 1939, on Arctostaphylos sp. (manzanita) (H. H. Keifer).

Quadraspidiotus juglans-regiae (Comstock), Greenspot, San Bernardino Co., June 27, 1955, on Juglans sp. (walnut) (G. M. Harper).

Quadraspidiotus perniciosus (Comstock), San Leandro, Alameda Co., May 26, 1954, on Prunus sp. (plum) (E. K. Strobridge).

Selenaspidus albus McKenzie, Norco, Riverside Co., June 28, 1954, on Euphorbia submammillaris (succulent) (R. M. Hawthorne and H. L. McKenzie).

\section{PLATE 3}

Chionaspis salicis-nigrae (Walsh), Alturas, Modoc Co., August 6, 1936, on Cornus sp. (dogwood).

Lepidosaphes beckii (Newman), Santa Barbara, December 16, 1954, on Citrus sinensis (orange) (W. S. Cummings).

Lepidosaphes ulmi (Linnaeus), Sacramento, November 5, 1953, on Salix sp. (willow) (H. H. Keifer).

Nilotaspis halli (Green), Chico, Butte Co., May 10, 1950, on Prunus amygdalus (almond) (E. F. Fosen).

Parlatoria camelliae (Comstock), Hickman, Stanislaus Co., February, 1954, on Camellia sp.

Parlatoria oleae (Colvée), Madera Co., January 7,1954 , on Olea europaea (olive) (T. B. Gallion).

Parlatoria pittospori Maskell, San Diego, December 9, 1954, on Pittosporum sp. (R. F. Wilkey).

Phenacaspis pinifoliae (Fitch), Crestline, San Bernardino Co., March 9, 1954, on Pinus ponderosa (yellow pine) (C. L. Downing).

Unaspis euonymi (Comstock), Sacramento, November 4, 1954, on Euonymus sp. (H. L. McKenzie). 


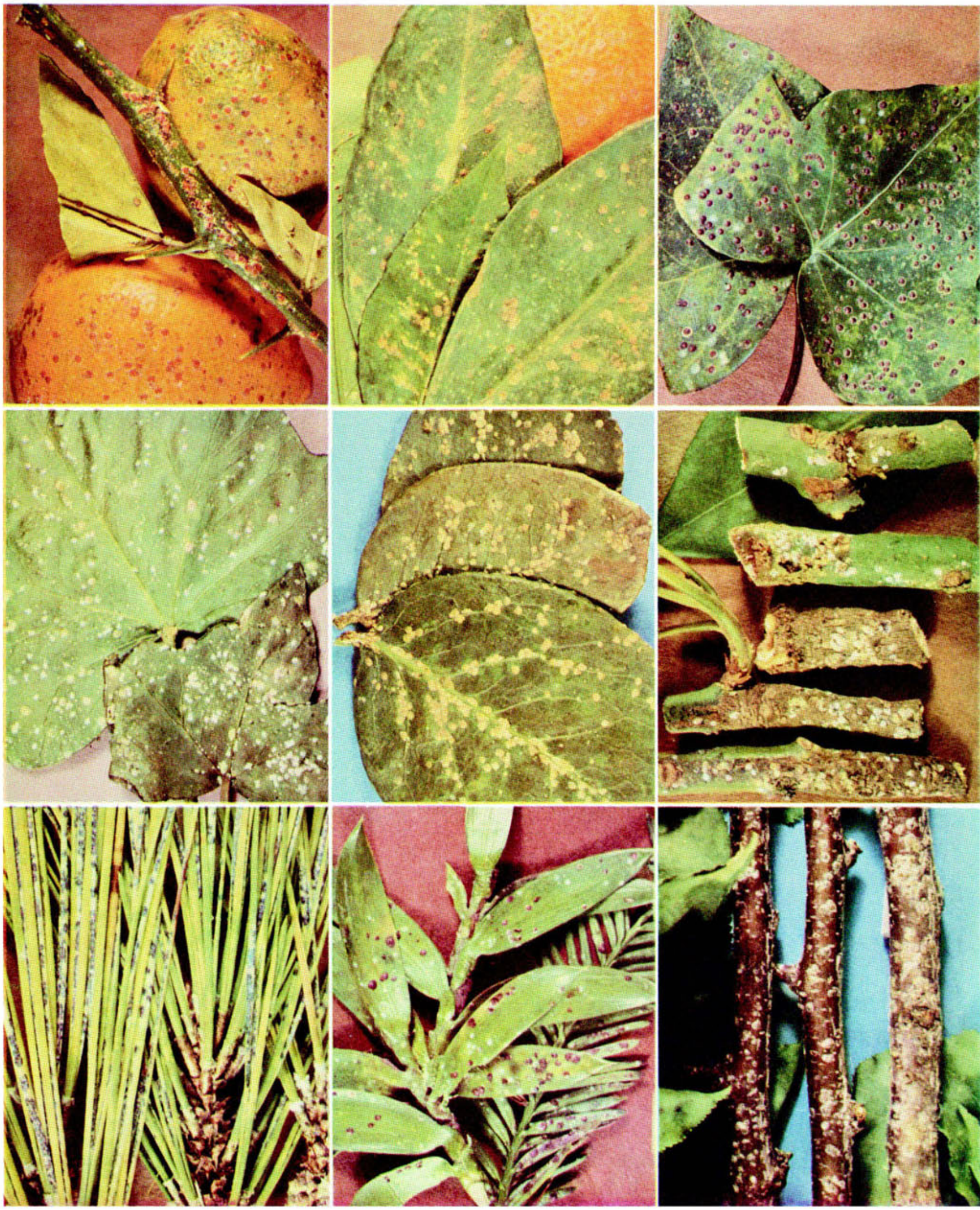

Top row. Left: Aonidiella aurantii (Maskell), California red scale; center: Aonidiella citrina (Coquillett), yellow scale; right: Chrysomphalus bifasciculatus Ferris, Bifasciculate scale.

Middle row. Left: Aspidiotus hederae (Vallot), ivy scale; center: Hemiberlesia degenerata (Leonardi), degenerate scale; right: Hemiberlesia lataniae (Signoret), Latania scale.

Bottom row. Left: Nuculaspis californica (Coleman), black pine leaf scale; center: Lindingaspis rossi (Maskell), black araucaria scale; right: Diaspidiotus ancylus (Putnam), Putnam scale. 
PLATE 2
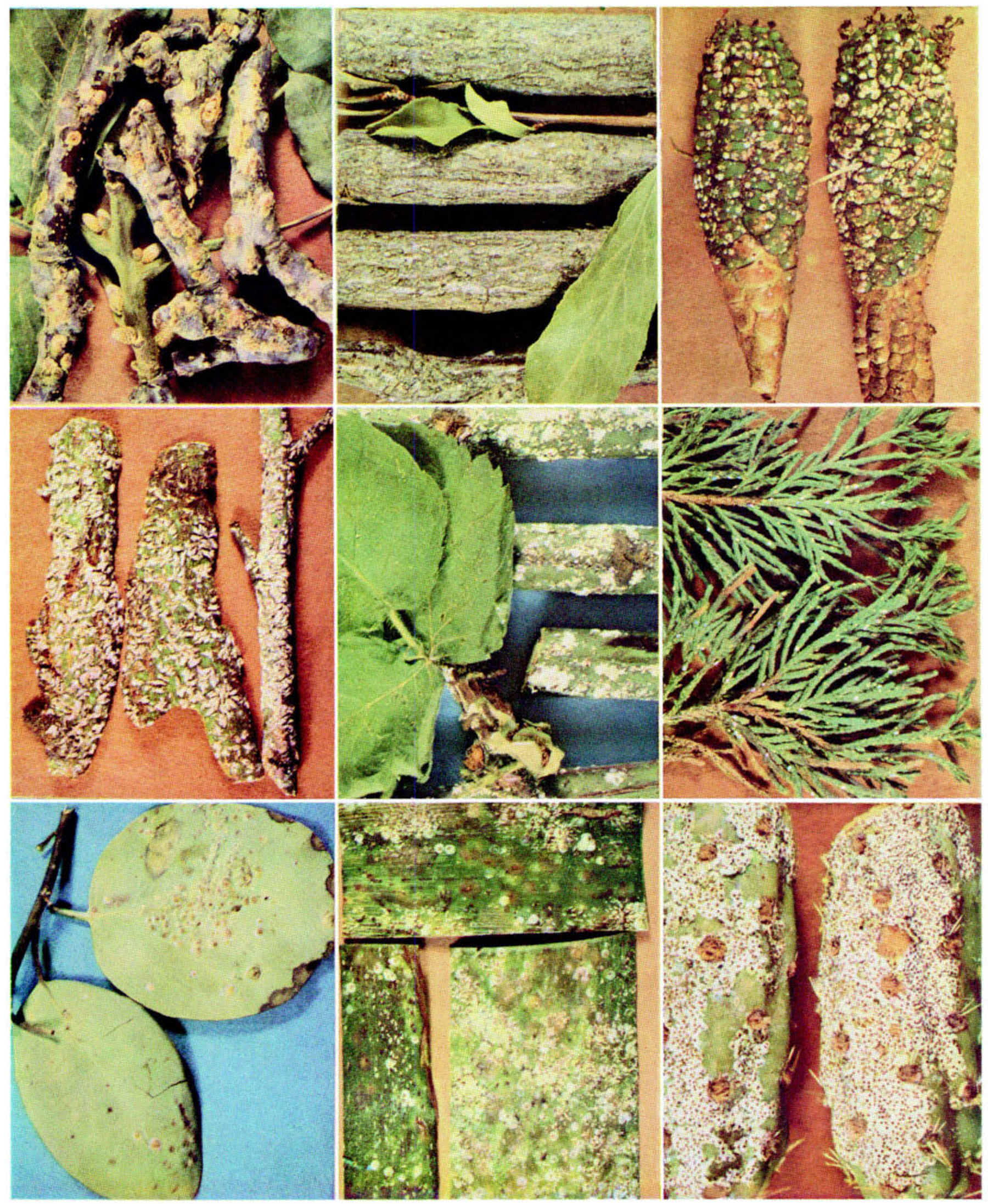

Top row. Left: Quadraspidiotus juglans-regiae (Comstock), walnut scale; center: Quadraspidiotus perniciosus (Comstock), San Jose scale; right: Selenaspidus albus McKenzie, white euphorbia scale.

Middle row. Left: Aonidomytilus ceanothi (Ferris), Ceanothus scale; center: Aulacaspis rosae (Bouché), rose scale; right: Carulaspis visci (Schrank), juniper scale.

Bottom row. Left: Diaspis manzanitae (Whitney), manzanita scale; center: Diaspis bromeliae (Kerner), pineapple scale; right: Diaspis erhinorarti (Rourhól rartus scale. 


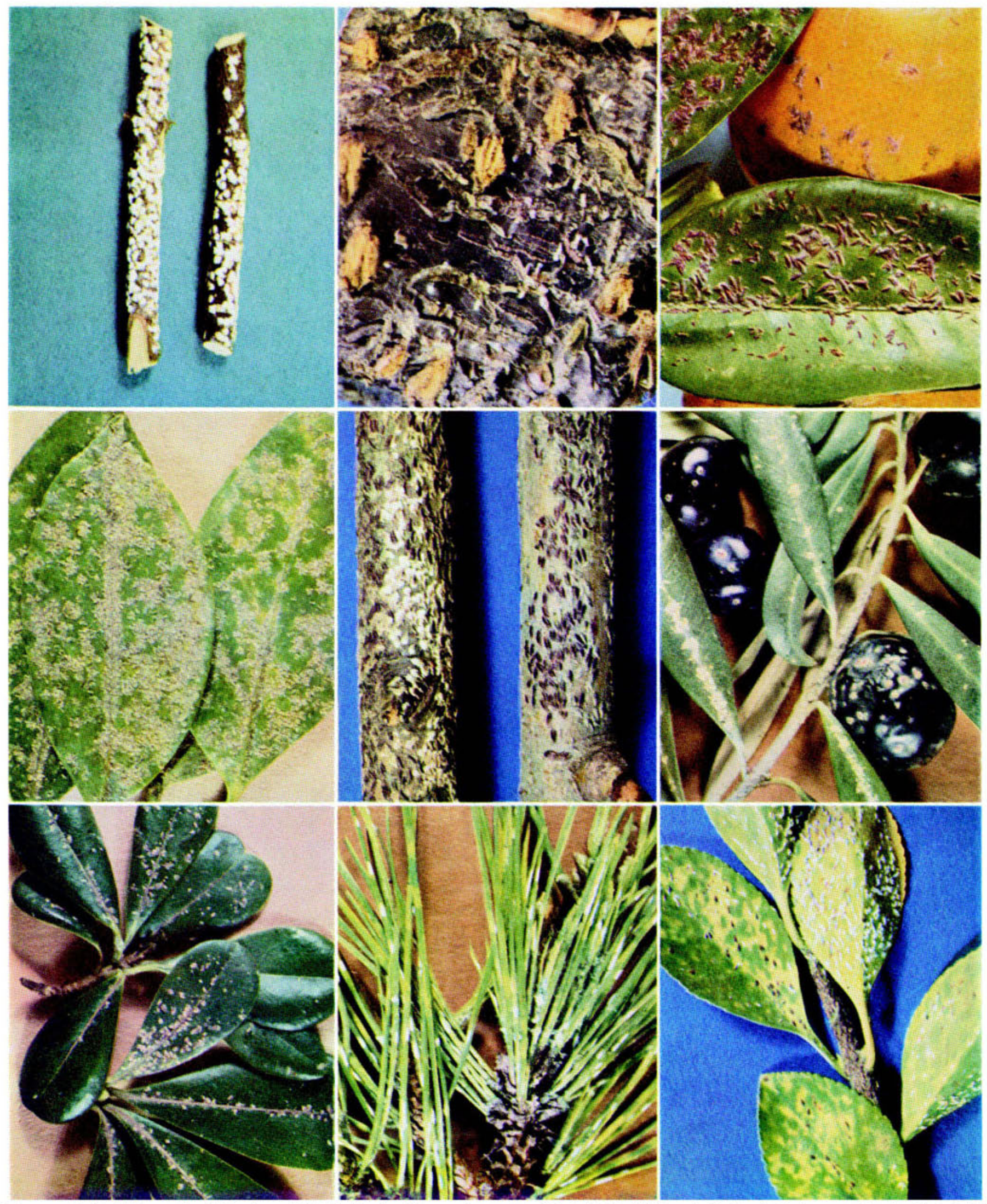

Top row. Left: Chionaspis salicis-nigrae (Walsh), black willow scale; center: Nilotaspis halli (Green), Hall scale (enlargement x3); right: Lepidosaphes beckii (Newman), purple scale.

Middle row. Left: Parlatoria camelliae Comstock, camellia parlatoria scale; center: Lepidosaphes ulmi (Linnaeus), oystershell scale; right: Parlatoria oleae (Colvée), olive parlatoria scale.

Bottom row. Left: Parlatoria pittospori Maskell, Pittosporum diaspidid; center: Phenacaspis pinifoliae (Fitch), pine needle scale; right: Unaspis euonymi (Comstock), Euonymus scale. 